\title{
On the Connection between Turbulent Motions and Particle Acceleration in Galaxy Clusters
}

\author{
D. Eckert ${ }^{1}$, M. Gaspari ${ }^{2,8}$, F. Vazza $^{3}$, F. Gastaldello ${ }^{4}$, A. Tramacere ${ }^{1}$, S. Zimmer ${ }^{5}$, S. Ettori ${ }^{6,7}$, and S. Paltani ${ }^{1}$ \\ ${ }^{1}$ Department of Astronomy, University of Geneva, 16, ch. d'Ecogia, CH-1290 Versoix, Switzerland; Dominique.Eckert@unige.ch \\ ${ }^{2}$ Department of Astrophysical Sciences, Princeton University, 4 Ivy Lane, Princeton, NJ 08544-1001, USA \\ INAF-Istituto di Radioastronomia, via Gobetti 101, I-40129 Bologna, Italy \\ ${ }^{4}$ INAF-IASF-Milano, via E. Bassini 15, I-20133 Milano, Italy \\ ${ }^{5}$ DPNC, University of Geneva, 24, Quai Ernest-Ansermet, CH-1211 Geneva, Switzerland \\ ${ }^{6}$ INAF-Osservatorio Astronomico di Bologna, via Pietro Gobetti 93/3, I-40129 Bologna, Italy \\ 7 INFN, Sezione di Bologna, viale Berti Pichat 6/2, I-40127 Bologna, Italy \\ Received 2017 May 5; revised 2017 June 14; accepted 2017 June 26; published 2017 July 12
}

\begin{abstract}
Giant radio halos are megaparsec-scale diffuse radio sources associated with the central regions of galaxy clusters. The most promising scenario to explain the origin of these sources is that of turbulent re-acceleration, in which $\mathrm{MeV}$ electrons injected throughout the formation history of galaxy clusters are accelerated to higher energies by turbulent motions mostly induced by cluster mergers. In this Letter, we use the amplitude of density fluctuations in the intracluster medium as a proxy for the turbulent velocity and apply this technique to a sample of 51 clusters with available radio data. Our results indicate a segregation in the turbulent velocity of radio halo and radio quiet clusters, with the turbulent velocity of the former being on average higher by about a factor of two. The velocity dispersion recovered with this technique correlates with the measured radio power through the relation $P_{\text {radio }} \propto \sigma_{v}^{3.3 \pm 0.7}$, which implies that the radio power is nearly proportional to the turbulent energy rate. In case turbulence cascades without being dissipated down to the particle acceleration scales, our results provide an observational confirmation of a key prediction of the turbulent re-acceleration model and possibly shed light on the origin of radio halos.
\end{abstract}

Key words: acceleration of particles - galaxies: clusters: general - radiation mechanisms: non-thermal radio continuum: general - turbulence

\section{Introduction}

Giant radio halos are megaparsec-scale diffuse radio sources associated with the central regions of galaxy clusters (see Feretti et al. 2012 for a review). Radio halos are a transient phenomenon observed in only a fraction of galaxy clusters (e.g., Venturi et al. 2007, 2008; Basu 2012), and recent studies have shown that they occur only in dynamically disturbed systems (Buote 2001; Cassano et al. 2010; Rossetti et al. 2011), suggesting a connection between particle acceleration and major cluster mergers.

The kinetic energy injected during major mergers first generates a turbulent cascade down to small spatial scales (Dolag et al. 2005; Vazza et al. 2009, 2017; Miniati 2015), which may ultimately be dissipated into gas heating, magnetic field amplification, and cosmic-ray acceleration. Stochastic acceleration generated by turbulent motions in the intracluster medium (ICM) is now thought to be the most plausible particle acceleration mechanism to re-energize a pre-existing population of $\sim \mathrm{MeV}$ electrons and explain the origin of radio halos (e.g., Brunetti et al. 2001; Petrosian \& Bykov 2008; Brunetti \& Lazarian 2011, 2016). The turbulent re-acceleration model reproduces a number of observed features, such as the existence of steep-spectrum radio halos (Brunetti et al. 2008; Macario et al. 2010), the curvature of the spectra at high frequency (Thierbach et al. 2003), and the link between radio halos and cluster mergers (Cassano et al. 2010). However, the connection between radio halos and ICM turbulence remains untested.

\footnotetext{
${ }^{8}$ Einstein and Spitzer fellow.
}

In this Letter, we use the power spectrum of X-ray surfacebrightness fluctuations in the ICM to search for a connection between turbulent motions and radio properties in galaxy clusters. Recent theoretical progress (Schuecker et al. 2004; Churazov et al. 2012; Gaspari \& Churazov 2013; Gaspari et al. 2014; Zhuravleva et al. 2014) has shown that gas density fluctuations act as a passive tracer of velocity fluctuations in the ICM and that the maximal amplitude of density fluctuations is linearly related to the turbulent Mach number. This method has been successfully applied to several clusters thus far (Zhuravleva et al. 2015; Arévalo et al. 2016; Hofmann et al. 2016; Khatri \& Gaspari 2016) and it was found to reproduce the level of turbulence directly measured in the Perseus cluster by Hitomi (Hitomi Collaboration et al. 2016). We retrieve the amplitude of density fluctuations at a fixed spatial scale and use this quantity as a proxy for the expected level of turbulence. We then search for a connection between turbulence and radio emission. The Letter is organized as follows. In Section 2, we present the sample and describe the methodology adopted to analyze the X-ray data. In Section 3, we present our results and discuss them in the framework of the turbulent re-acceleration model in Section 4. In Section 5, we summarize our main conclusions.

\section{Analysis}

\subsection{The Sample}

We base our analysis on the sample of 55 clusters with available radio information at the nearly uniform depth from Cassano et al. (2013). The sample is based on the GMRT radio halo survey (Venturi et al. 2007, 2008) with the addition of 
clusters with known radio emission from the literature. We searched the XMM-Newton, Chandra, and ROSAT/PSPC archives for available X-ray data and selected a subsample of 51 clusters for which the quality of the X-ray data is sufficient to retrieve the level of surface-brightness fluctuation over large spatial scales. A radio halo was detected for 25 clusters in our sample, whereas for the remaining 26 systems, upper limits to the radio flux at $1.4 \mathrm{GHz}$ are available. For nearby clusters $(z<0.1)$, we use ROSAT/PSPC, as the wide field of view (FOV) of this instrument allows us to cover uniformly a circular region of 1 $\mathrm{Mpc}$ radius, i.e., comparable to the typical sizes of radio halos. In the redshift range $0.1<z<0.3$, we use XMM-Newton as our instrument of choice given its large collecting area and FOV, with the exception of a few cases (e.g., Bullet, A520) for which deep Chandra data are available. Beyond $z=0.3$ only the angular resolution of Chandra is sufficient to resolve scales less than $\sim 50 \mathrm{kpc}$; thus, we restrict ourselves to Chandra data. The final sample is presented in Table 1 together with its relevant properties and the adopted instrument. In all cases, we restrict ourselves to the [0.5-2] keV band to be sensitive only to density fluctuations. Given that our sample comprises only hot clusters, this choice of energy band is appropriate.

\subsection{Data Reduction}

\subsubsection{XMM-Newton}

We reduced the XMM-Newton data using XMMSAS v15.0 and the ESAS software package (Snowden et al. 2008). Time periods including flaring soft proton flux are filtered out to extract clean event files. We use the unexposed corners of the CCD chips to measure the quiescent background level in each observation. We then renormalize the filter-wheel-closed data sets to match the count rates measured in the CCD corners, which allows us to create model particle background images for each observation. We extract photon images and exposure maps in the [0.5-2] keV band from the cleaned event files. Finally, to avoid contamination from point sources, we run ewavelet in each observation and mask a circular region of 30 arcsec radius around each point source. For more details we refer the reader to Eckert et al. (2014).

\subsubsection{Chandra}

We analyzed the data using the CIAO v4.9 software package and CALDB v4.7.3. For each observation, the raw data are reprocessed with the latest calibration files by running the chandra_repro pipeline. Periods of flaring background are removed by using the deflare tool. We then extract photon images and exposure maps in the [0.5-2] keV band. To estimate the local background, we use the blanksky and blanksky_image tools (Hickox \& Markevitch 2006) to estimate the count rate in the [9.5-12] keV band and renormalize blank-sky data sets to the appropriate level for each observation. Point sources are detected using wavdetect and masked during the analysis.

\subsubsection{ROSAT/PSPC}

We reduced the ROSAT data using the Extended Source Analysis Software package (Snowden et al. 1994). The analysis pipeline follows exactly the method presented in Eckert et al. (2012). We create photon images, exposure maps, and background in the ROSAT R37 band (corresponding to $[0.42-2.01] \mathrm{keV})$ and mask the detected point sources according to the size of the PSF at each radius. For more details on the analysis procedure we refer to Eckert et al. (2012).

\subsection{Temperature Measurements}

We extracted mean spectroscopic temperatures within the same region of $1 \mathrm{Mpc}$ radius as for the extraction of the amplitude of surface-brightness fluctuations. For XMM-Newton, we follow the method outlined in Eckert et al. (2014). Briefly, we use a phenomenological model to describe the spectral shape of the non X-ray background, which we fit together with the source. The sky background is modeled as the sum of two APEC models (Smith et al. 2001) for the Galactic foregrounds and an absorbed power law for the cosmic X-ray background. In the case of Chandra, we use an offset region to describe the local background. In both cases, the source is described as a singletemperature APEC model absorbed by the Galactic $N_{H}$, which we fix to the $21 \mathrm{~cm}$ value (Kalberla et al. 2005). The spectra are then fit in XSPEC using C statistic (Cash 1979). The best-fit spectroscopic temperatures are reported in Table 1.

\subsection{Surface-brightness Fluctuations}

To compute the amplitude of density fluctuations, we create a two-dimensional model for the large-scale gas distribution and extract a residual map by dividing the observed emission by the model brightness. The amplitude of surface-brightness fluctuations is then recovered from the Fourier power spectrum of the fluctuations in the residual image at the desired scale.

To model the cluster gas distribution, we assume that the morphology of the cluster can be described by an elliptical beta model (Cavaliere \& Fusco-Femiano 1976). We use a weighted principal component analysis method to determine the centroid of emission and the ellipticity parameters (major and minor axes, rotation angle). We mask obvious substructures associated with individual subclumps (e.g., the Bullet in $1 \mathrm{E}$ 0657-56) to avoid introducing additional power unrelated to turbulent motions. We then extract a surface-brightness profile using PROFFIT (Eckert et al. 2011), and we fit the beta model to the data using $\mathrm{C}$ statistic. In the cases where a double beta model is statistically favored, we adopt the double beta model solution as our model of choice. We then create a model image for the two-dimensional gas distribution by folding the best-fit model with the exposure map and adding the background map.

To compute the Fourier power spectrum at the desired scale, we use the modified $\Delta$-variance method introduced by Arévalo et al. (2012), which allows us to take holes and non-periodic boxes into account. In this method, the image and the mask are convolved with a Mexican hat filter, and the filtered image is corrected for the spurious features introduced by holes. The variance of the filtered image is proportional to the power at the chosen scale. To estimate the noise level, we simulate Poisson noise on top of the model image and apply the same procedure. The variance of the noise image is then subtracted from that of the true image. The uncertainty in the measurement of the power spectrum is estimated by splitting the filtered image into 20 subregions, computing the power in each region separately, and performing $10^{4}$ bootstrap resamplings of the measured values. We then adopt the standard deviation of the bootstrap distribution as our $1 \sigma$ error. 
Table 1

Properties of the Cluster Sample ${ }^{\mathrm{a}}$

\begin{tabular}{|c|c|c|c|c|c|c|c|}
\hline Cluster & $z$ & $k T_{1 \mathrm{Mpc}}[\mathrm{keV}]$ & $P_{1.4}\left[\mathrm{~W} \mathrm{~Hz}^{-1}\right]$ & $A_{2 D}$ & $\mathrm{Mach}_{1 \mathrm{D}}$ & $\sigma_{v}\left[\mathrm{~km} \mathrm{~s}^{-1}\right]$ & Instrument \\
\hline \multicolumn{8}{|l|}{ Upper Limits } \\
\hline A2697 & 0.232 & $6.94 \pm 0.14$ & $<0.41$ & $0.06 \pm 0.01$ & $0.12 \pm 0.01$ & $250 \pm 27$ & $\mathrm{X}$ \\
\hline A141 & 0.23 & $5.9 \pm 0.15$ & $<0.43$ & $0.08 \pm 0.01$ & $0.16 \pm 0.02$ & $325 \pm 38$ & $\mathrm{X}$ \\
\hline A3088 & 0.2537 & $6.42 \pm 0.16$ & $<0.43$ & $0.07 \pm 0.01$ & $0.14 \pm 0.02$ & $291 \pm 39$ & $\mathrm{X}$ \\
\hline RXCJ0437.1+0043 & 0.285 & $6.45 \pm 0.2$ & $<0.65$ & $0.04 \pm 0.01$ & $0.09 \pm 0.02$ & $179 \pm 39$ & $\mathrm{X}$ \\
\hline RXCJ1115.8+0129 & 0.3499 & $6.21 \pm 0.11$ & $<0.47$ & $0.06 \pm 0.01$ & $0.11 \pm 0.02$ & $227 \pm 41$ & $\mathrm{X}$ \\
\hline A2631 & 0.2779 & $7.61 \pm 0.3$ & $<0.41$ & $0.09 \pm 0.01$ & $0.16 \pm 0.02$ & $374 \pm 48$ & $\mathrm{X}$ \\
\hline A2645 & 0.251 & $6.28 \pm 0.18$ & $<0.59$ & $0.08 \pm 0.01$ & $0.15 \pm 0.01$ & $315 \pm 25$ & $\mathrm{X}$ \\
\hline A2667 & 0.2264 & $5.99 \pm 0.08$ & $<0.45$ & $0.08 \pm 0.01$ & $0.15 \pm 0.01$ & $310 \pm 24$ & $\mathrm{X}$ \\
\hline Z348 & 0.2537 & $2.94 \pm 0.05$ & $<0.65$ & $0.05 \pm 0.01$ & $0.10 \pm 0.02$ & $144 \pm 22$ & $\mathrm{X}$ \\
\hline RXJ0142.0+2131 & 0.2803 & $7.37 \pm 0.58$ & $<0.45$ & $0.09 \pm 0.02$ & $0.18 \pm 0.03$ & $402 \pm 70$ & $\mathrm{C}$ \\
\hline A267 & 0.231 & $5.64 \pm 0.17$ & $<0.34$ & $0.08 \pm 0.01$ & $0.16 \pm 0.01$ & $306 \pm 27$ & $\mathrm{X}$ \\
\hline RXJ0439.0+0715 & 0.23 & $6.23 \pm 0.48$ & $<0.46$ & $0.07 \pm 0.02$ & $0.13 \pm 0.04$ & $272 \pm 77$ & $\mathrm{C}$ \\
\hline RXJ0439.0+0520 & 0.208 & $6.53 \pm 0.25$ & $<0.32$ & $0.01 \pm 0.04$ & $0.02 \pm 0.08$ & $41 \pm 167$ & $\mathrm{C}$ \\
\hline A611 & 0.288 & $6.63 \pm 0.45$ & $<0.43$ & $0.07 \pm 0.03$ & $0.13 \pm 0.05$ & $273 \pm 105$ & $\mathrm{X}$ \\
\hline Z2089 & 0.2347 & $3.82 \pm 0.13$ & $<0.26$ & $0.07 \pm 0.01$ & $0.13 \pm 0.01$ & $205 \pm 16$ & $\mathrm{X}$ \\
\hline A781 & 0.2984 & $6.21 \pm 0.12$ & $<0.36$ & $0.11 \pm 0.01$ & $0.22 \pm 0.02$ & $457 \pm 42$ & $\mathrm{X}$ \\
\hline Z2701 & 0.214 & $5.41 \pm 0.22$ & $<0.35$ & $0.07 \pm 0.01$ & $0.13 \pm 0.02$ & $250 \pm 42$ & $\mathrm{C}$ \\
\hline A1423 & 0.213 & $7.65 \pm 0.39$ & $<0.38$ & $0.07 \pm 0.01$ & $0.13 \pm 0.01$ & $306 \pm 32$ & $\mathrm{C}$ \\
\hline A1576 & 0.279 & $7.73 \pm 0.46$ & $<0.64$ & $0.10 \pm 0.02$ & $0.19 \pm 0.03$ & $429 \pm 69$ & $\mathrm{C}$ \\
\hline RXJ1532.9+3021 & 0.345 & $4.89 \pm 0.07$ & $<0.66$ & $0.04 \pm 0.00$ & $0.07 \pm 0.01$ & $128 \pm 15$ & $\mathrm{X}$ \\
\hline A2146 & 0.2343 & $6.59 \pm 0.17$ & $<0.39$ & $0.08 \pm 0.01$ & $0.15 \pm 0.02$ & $319 \pm 35$ & $\mathrm{C}$ \\
\hline A2261 & 0.224 & $8.01 \pm 0.31$ & $<0.32$ & $0.08 \pm 0.01$ & $0.15 \pm 0.02$ & $357 \pm 42$ & $\mathrm{X}$ \\
\hline A2537 & 0.2966 & $7.46 \pm 0.34$ & $<0.51$ & $0.06 \pm 0.02$ & $0.12 \pm 0.03$ & $263 \pm 78$ & $\mathrm{X}$ \\
\hline RXJ0027.6+2616 & 0.3649 & $5.29 \pm 0.58$ & $<0.74$ & $0.08 \pm 0.01$ & $0.15 \pm 0.03$ & $284 \pm 53$ & $\mathrm{X}$ \\
\hline Z5768 & 0.266 & $3.34 \pm 0.23$ & $<0.36$ & $0.09 \pm 0.01$ & $0.17 \pm 0.02$ & $260 \pm 35$ & $\mathrm{X}$ \\
\hline S780 & 0.2357 & $6.67 \pm 0.22$ & $<0.38$ & $0.09 \pm 0.01$ & $0.17 \pm 0.01$ & $354 \pm 28$ & $\mathrm{C}$ \\
\hline \multicolumn{8}{|l|}{ Radio Halos } \\
\hline A2744 & 0.307 & $8.69 \pm 0.32$ & $18.62 \pm 0.94$ & $0.14 \pm 0.02$ & $0.26 \pm 0.04$ & $629 \pm 89$ & $\mathrm{C}$ \\
\hline A209 & 0.206 & $6.67 \pm 0.12$ & $1.99 \pm 0.21$ & $0.07 \pm 0.00$ & $0.13 \pm 0.01$ & $273 \pm 15$ & $\mathrm{X}$ \\
\hline A2163 & 0.203 & $15.22 \pm 0.16$ & $22.91 \pm 1.16$ & $0.12 \pm 0.01$ & $0.22 \pm 0.02$ & $711 \pm 65$ & $\mathrm{X}$ \\
\hline RXCJ2003.5-2323 & 0.3171 & $9.35 \pm 0.53$ & $10.71 \pm 1.73$ & $0.13 \pm 0.01$ & $0.24 \pm 0.02$ & $603 \pm 45$ & $\mathrm{C}$ \\
\hline A520 & 0.199 & $7.21 \pm 0.16$ & $2.45 \pm 0.18$ & $0.13 \pm 0.01$ & $0.24 \pm 0.02$ & $536 \pm 55$ & $\mathrm{C}$ \\
\hline A773 & 0.217 & $7.65 \pm 0.19$ & $1.48 \pm 0.16$ & $0.10 \pm 0.01$ & $0.20 \pm 0.02$ & $451 \pm 35$ & $\mathrm{X}$ \\
\hline A1758a & 0.28 & $7.22 \pm 0.17$ & $5.75 \pm 0.98$ & $0.14 \pm 0.02$ & $0.27 \pm 0.03$ & $589 \pm 65$ & $\mathrm{C}$ \\
\hline A2219 & 0.2281 & $10.02 \pm 0.25$ & $5.63 \pm 0.8$ & $0.09 \pm 0.01$ & $0.17 \pm 0.02$ & $432 \pm 40$ & $\mathrm{X}$ \\
\hline A521 & 0.2475 & $6.41 \pm 0.21$ & $1.45 \pm 0.13$ & $0.12 \pm 0.01$ & $0.22 \pm 0.02$ & $462 \pm 44$ & $\mathrm{C}$ \\
\hline A697 & 0.282 & $9.66 \pm 0.85$ & $1.51 \pm 0.14$ & $0.09 \pm 0.02$ & $0.18 \pm 0.03$ & $458 \pm 81$ & $\mathrm{X}$ \\
\hline A1300 & 0.3075 & $7.85 \pm 0.25$ & $3.8 \pm 1.43$ & $0.14 \pm 0.01$ & $0.26 \pm 0.03$ & $595 \pm 62$ & $\mathrm{C}$ \\
\hline CL0016+16 & 0.541 & $9.42 \pm 0.3$ & $5.01 \pm 0.31$ & $0.10 \pm 0.01$ & $0.19 \pm 0.01$ & $472 \pm 35$ & $\mathrm{C}$ \\
\hline A1914 & 0.1712 & $11.38 \pm 0.67$ & $5.62 \pm 0.43$ & $0.12 \pm 0.01$ & $0.24 \pm 0.02$ & $657 \pm 53$ & $\mathrm{C}$ \\
\hline A665 & 0.1819 & $7.53 \pm 0.17$ & $2.51 \pm 0.21$ & $0.12 \pm 0.01$ & $0.24 \pm 0.02$ & $539 \pm 39$ & $\mathrm{X}$ \\
\hline A545 & 0.154 & $6.57 \pm 0.09$ & $1.41 \pm 0.22$ & $0.10 \pm 0.01$ & $0.19 \pm 0.01$ & $399 \pm 24$ & $\mathrm{X}$ \\
\hline Coma & 0.0231 & $8.28 \pm 0.13$ & $0.76 \pm 0.06$ & $0.09 \pm 0.01$ & $0.18 \pm 0.02$ & $420 \pm 45$ & $\mathrm{R}$ \\
\hline A2256 & 0.0581 & $7.65 \pm 0.63$ & $0.85 \pm 0.08$ & $0.13 \pm 0.01$ & $0.25 \pm 0.02$ & $571 \pm 56$ & $\mathrm{R}$ \\
\hline Bullet & 0.296 & $14.58 \pm 0.4$ & $23.44 \pm 1.51$ & $0.17 \pm 0.02$ & $0.31 \pm 0.03$ & $977 \pm 99$ & $\mathrm{C}$ \\
\hline A2255 & 0.0806 & $5.81 \pm 0.2$ & $0.81 \pm 0.17$ & $0.09 \pm 0.01$ & $0.18 \pm 0.01$ & $365 \pm 22$ & $\mathrm{R}$ \\
\hline A2319 & 0.0557 & $9.6 \pm 0.3$ & $2.45 \pm 0.19$ & $0.11 \pm 0.01$ & $0.21 \pm 0.02$ & $529 \pm 46$ & $\mathrm{R}$ \\
\hline MACSJ0717.5+3745 & 0.548 & $13.59 \pm 0.68$ & $52.48 \pm 20.56$ & $0.21 \pm 0.02$ & $0.40 \pm 0.03$ & $1206 \pm 96$ & $\mathrm{C}$ \\
\hline A1995 & 0.3186 & $7.58 \pm 0.41$ & $1.66 \pm 0.23$ & $0.08 \pm 0.01$ & $0.14 \pm 0.03$ & $326 \pm 59$ & $\mathrm{C}$ \\
\hline MACSJ1149.5+2223 & 0.544 & $13.14 \pm 0.9$ & $2.29 \pm 0.95$ & $0.15 \pm 0.02$ & $0.29 \pm 0.03$ & $853 \pm 94$ & $\mathrm{C}$ \\
\hline PLCKG171.9-40.7 & 0.27 & $12.78 \pm 0.79$ & $4.9 \pm 1.35$ & $0.08 \pm 0.01$ & $0.15 \pm 0.01$ & $438 \pm 40$ & $\mathrm{X}$ \\
\hline A754 & 0.0542 & $8.33 \pm 0.05$ & $0.63 \pm 0.07$ & $0.15 \pm 0.01$ & $0.28 \pm 0.02$ & $676 \pm 46$ & $\mathrm{R}$ \\
\hline
\end{tabular}

Note.

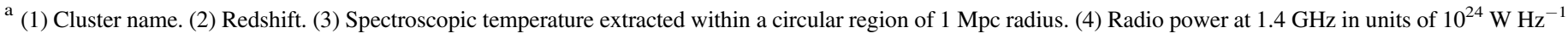

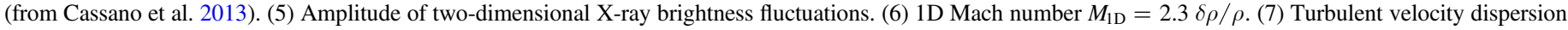
$\sigma_{v}=M_{3 \mathrm{D}} c_{\mathrm{s}}$, with $c_{\mathrm{s}} \approx\left(\gamma k T_{1 \mathrm{Mpc}} / \mu m_{p}\right)^{0.5}$. (8) X-ray instrument used for the analysis $(\mathrm{X}=$ XMM-Newton, C $=$ Chandra, $\mathrm{R}=$ ROSAT).

\subsection{Turbulent Velocity}

As shown by Arévalo et al. (2012), filtering the image at a given scale $\ell$ allows us to select fluctuations at a wavenumber $k=\frac{1}{\sqrt{2 \pi^{2}} \ell}$. For the present work, we choose a smoothing scale $\ell=150 \mathrm{kpc}$, corresponding to $k^{-1}=660 \mathrm{kpc}$. Such a scale roughly corresponds to the typical size of groups accreting onto 

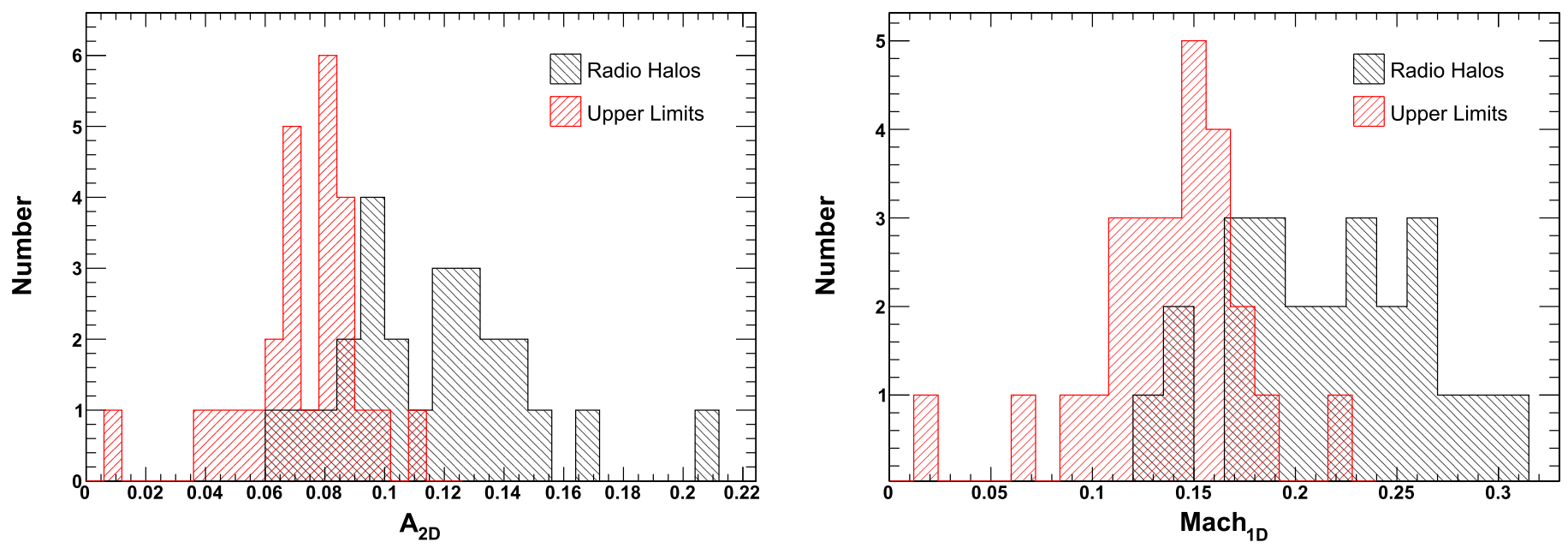

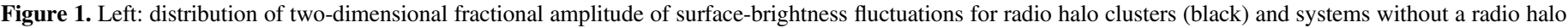
(red). Right: same as in the left panel, but for the 1D turbulent Mach number estimated from the deprojected density fluctuations.

clusters, i.e., it should be close to the injection scale of turbulent motions, at which the amplitude of fluctuations is expected to peak. Finally, the projected two-dimensional amplitude is converted into three-dimensional fluctuations $\delta \rho / \rho$ by using the best-fit beta model parameters and computing numerically the power induced by the projection of the emissivity distribution along the line of sight (see Equation (11) of Churazov et al. 2012). For consistency, we checked that the amplitudes recovered from Chandra and $X M M-N e w t o n$ data are in agreement when data from both telescopes are available. We found an excellent agreement between the two X-ray telescopes, giving us confidence that our method is robust.

As shown in Gaspari \& Churazov (2013), in the subsonic regime the maximum amplitude of density fluctuations is linearly related to the turbulent Mach number $M=\frac{\sigma_{v}}{c_{s}}$, with $\sigma_{v}$ the turbulent velocity dispersion and $c_{s}$ the sound speed in the medium. The relation reads $M_{1 \mathrm{D}} \approx 2.3 \frac{\delta \rho}{\rho}$ or $M_{3 \mathrm{D}} \approx$ $4 \frac{\delta \rho}{\rho}(L / 500 \mathrm{kpc})^{-0.25} \approx 3.7 \frac{\delta \rho}{\rho}$ for our choice of scale. The intrinsic scatter of the relation is expected to be $\sim 30 \%$ (Zhuravleva et al. 2014). For each cluster, we compute the average spectroscopic temperature within the same circular region of $1 \mathrm{Mpc}$ radius to estimate the average sound speed $c_{s}=\left(\gamma k T / \mu m_{p}\right)^{1 / 2}$ with $\gamma=5 / 3$ and convert the Mach number into turbulent velocity.

\section{Results}

\subsection{Amplitude of Surface-brightness Fluctuations}

We estimated the two-dimensional amplitude of surfacebrightness fluctuations $A_{2 D}$ at a fixed scale $k^{-1}=660 \mathrm{kpc}$ and the corresponding Mach number and turbulent velocity dispersion for all clusters in our sample. The resulting values are provided in Table 1. In the left-hand panel of Figure 1, we show the distribution of the values of $A_{2 D}(k=1 / 660 \mathrm{kpc})$ split between the radio halo and non-radio halo populations. We see that the radio halo clusters exhibit on average a higher value of $A_{2 D}$ than the upper limit population, with mean values $A_{2 D, R H}=0.117$ and $A_{2 D, U L}=0.072$ for the radio halo and upper limit populations, respectively. We performed a Kolmogorov-Smirnov (K-S) test to determine the probability that the two sets of values are drawn from a common parent population. The K-S test returns a probability of $4 \times 10^{-6}$, meaning that the two distributions are different at $4.6 \sigma$ confidence. If instead we focus only on the high-mass objects $\left(M_{500}>5 \times 10^{14} M_{\odot}\right)$ for which the existing radio data should be deep enough to detect a radio halo, the K-S probability reduces to $6 \times 10^{-5}(4.0 \sigma)$.

Using the deprojection factors estimated in Section 2.5, we then computed the amplitudes of 3D fluctuations of $\delta \rho / \rho$, which we converted into 1D Mach numbers using the relation $M_{1 \mathrm{D}} \approx 2.3 \delta \rho / \rho$ (Gaspari \& Churazov 2013). The distribution of the values of $M_{1 \mathrm{D}}$ is shown in the right-hand panel of Figure 1, again splitting the sample into the radio halo and radio quiet populations. A clear segregation is observed between the two populations, most radio halo clusters exhibiting a value of $M_{1 \mathrm{D}}$ in the range $0.15-0.3$ (which is consistent with the sample of Hofmann et al. 2016), whereas for the clusters where no radio emission was detected, the typical value of $M_{1 \mathrm{D}}$ is $\sim 0.12$ and always less than $\sim 0.2$. In this case, the K-S test returns a probability $p=2 \times 10^{-7}$ that the two data sets are drawn from the same parent distribution, i.e., the result is significant at $5.2 \sigma$.

\subsection{Correlation with Radio Power}

For each cluster, we used the average temperatures reported in Table 1 to determine the sound speed in the medium. We then estimated the average three-dimensional turbulent velocity dispersion through the relation $\sigma_{v}=M_{3 \mathrm{D}} c_{\mathrm{s}} \approx 3.7 c_{\mathrm{s}} \delta \rho / \rho$ (Gaspari \& Churazov 2013, Equation (22)). Note that our average temperatures are spectroscopic emission-weighted temperatures (e.g., Mazzotta et al. 2004), and thus our estimation of the sound speed does not exactly match the mean mass-weighted sound speed in the system. In Figure 2, we show the main result of the current study plotting the cluster $1.4 \mathrm{GHz}$ radio power versus the estimated turbulent velocity dispersion. Again, a segregation is observed between the radio halo and upper limit populations. For radio halo clusters, the radio power appears to correlate with the velocity dispersion, with the Pearson coefficient $\rho=0.80 \pm 0.02$ for 25 data points, indicating that the two quantities are significantly correlated. 


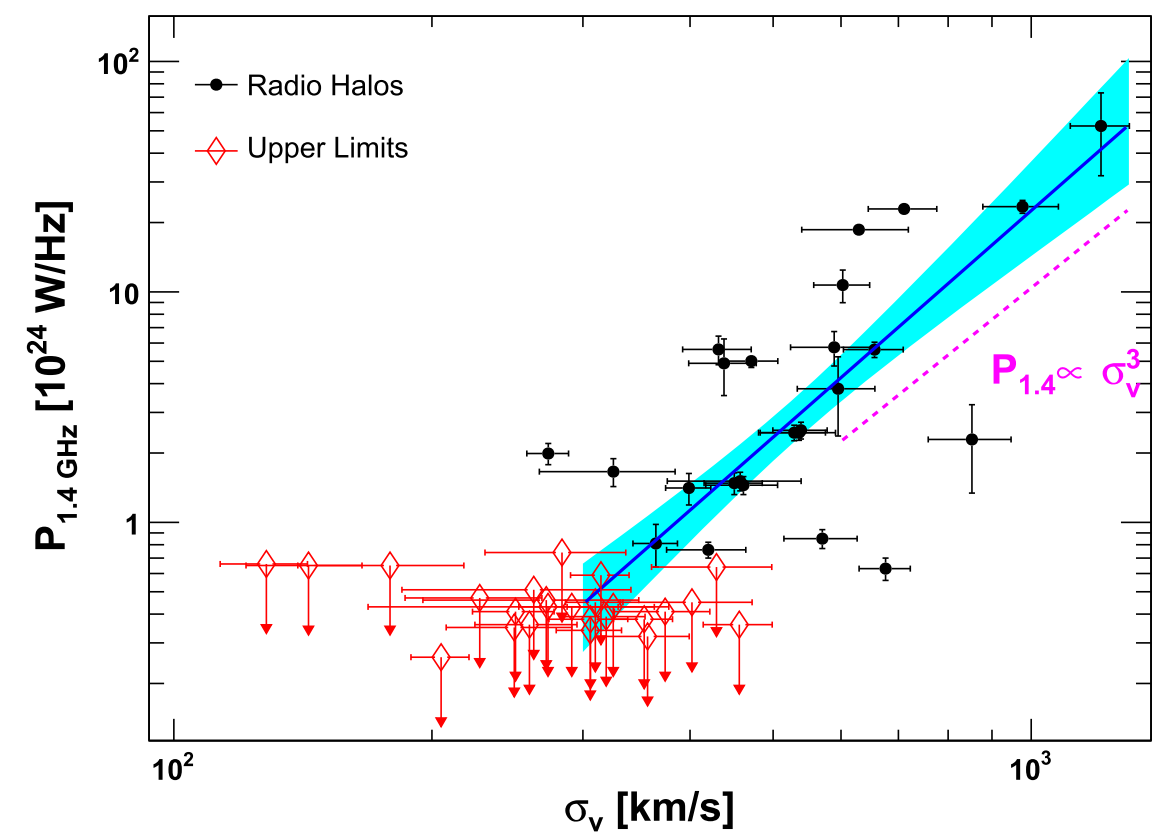

Figure 2. Radio power at $1.4 \mathrm{GHz}$ as a function of velocity dispersion $\sigma_{v}=M_{3 \mathrm{D}} c_{\mathrm{S}}$ for radio halo clusters (black) and radio upper limits (red). The blue curve and shaded area show the best fit to the data with a power law and its $1 \sigma$ error envelope.

To describe the relation between $\sigma_{v}$ and $P_{1.4}$, we fitted the data with a power law. Namely, we modeled the data as

$$
\log \left(\frac{P_{1.4 \mathrm{GHz}}}{10^{24} \mathrm{~W} \mathrm{~Hz}^{-1}}\right)=\log P_{0}+\alpha \log \left(\frac{\sigma_{v}}{500 \mathrm{~km} \mathrm{~s}^{-1}}\right) .
$$

We used the Bayesian routine linmix_err (Kelly 2007) to fit the data, taking both the data points and radio upper limits into account. The best-fitting values for the parameters are $\alpha=3.27_{-0.61}^{+0.71}$ and $P_{0}=2.34_{-0.49}^{+0.53}$, with an intrinsic scatter $\sigma_{\ln P \mid \sigma_{v}}=0.44_{-0.13}^{+0.18}$. We note that the relation between the projected surface-brightness fluctuations and three-dimensional velocity field should exhibit a substantial scatter; thus, the scatter of $\sim 40 \%$ observed here is not surprising. Note that the relation reported in Figure 2 includes a hidden correlation, as the radio power is a steep function of cluster mass (e.g., Cassano et al. 2013) and the sound speed $c_{s}$ also depends on cluster mass, albeit much more mildly.

\section{Discussion}

The main results of this work have potentially important implications on the origin of radio halos, which we discuss here. While previous works have found that radio halos arise almost only in morphologically disturbed systems (Buote 2001; Cassano et al. 2010, 2013; Cuciti et al. 2015), as evidenced, e.g., by their centroid shifts, our analysis shows that radio halo clusters remain on average more perturbed even after subtracting the large-scale gas distribution and masking substructures. We expect that surface-brightness fluctuations on top of the large-scale gas distribution trace residual gas motions. Numerical simulations predict that ICM turbulence should be the dominant source of residual motions and hence of ICM fluctuations (e.g., Lau et al. 2009; Vazza et al. 2009), although other sources of fluctuations (e.g., shocks, cold fronts, ram-pressure stripping) play a role to some extent. Our work thus establishes a connection between turbulent motions and particle acceleration in galaxy clusters, which corroborates a key prediction of the turbulent re-acceleration scenario (e.g., Brunetti et al. 2001).

In case the density fluctuations measured here can be entirely ascribed to turbulence, Figure 2 shows that the recovered turbulent velocity dispersion correlates with the radio power at $1.4 \mathrm{GHz}$, which can be interpreted in the framework of the turbulent re-acceleration scheme. The energy rate per unit volume related to turbulence can be estimated as

$$
\begin{aligned}
P_{\text {turb }} \approx & 9.8 \times 10^{-25}\left(\frac{\sigma_{v}}{500 \mathrm{~km} \mathrm{~s}^{-1}}\right)^{3}\left(\frac{n_{\text {gas }}}{10^{-2} \mathrm{~cm}^{-3}}\right) \\
& \times\left(\frac{L_{\text {inj }}}{500 \mathrm{kpc}}\right)^{-1} \mathrm{erg} \mathrm{s}^{-1} \mathrm{~cm}^{-3} .
\end{aligned}
$$

In the classical Kolmogorov model, the kinetic power across the cascade is preserved, regardless of the specific dissipation mechanism. While the specific scales and mechanisms for the ultimate dissipation of turbulent motions in the ICM are currently unknown (see, e.g., Brunetti \& Lazarian 2011), it is reasonable to assume that a fraction of the kinetic power through the cascade goes into the re-acceleration of radio emitting electrons. The exact relation between $P_{\text {radio }}$ and $P_{\text {turb }}$ is uncertain as it depends on many unknown factors. Yet, our data support (within $1 \sigma$ ) a simple $P_{\text {radio }} \propto \sigma_{v}^{3} \propto P_{\text {turb }}$, suggesting that $A_{2 D}$ can capture the bulk of the energy involved in the reacceleration process. However, the variety of mechanisms superseding the dissipation of plasma turbulence in the ICM (heating, magnetic field amplification, acceleration of cosmicray electrons and protons) is yet unknown; thus, the relation between the emitted power and the turbulent kinetic energy should be further investigated.

Although the segregation between radio halo and radio quiet clusters in Figures 1 and 2 is clear, there are obviously a number of outliers. Among these, we note the cases of A209 and A781. A209 is the radio halo cluster with the lowest values of $M_{1 \mathrm{D}}(0.13 \pm 0.01)$ and $\sigma_{v}\left(273 \pm 15 \mathrm{~km} \mathrm{~s}^{-1}\right)$. Such values 
would classify this object firmly within the "upper limit" quadrant. A209 could be a fading radio halo in which turbulence is now being dissipated. Alternatively, projection effects may hide large-scale fluctuations, which would lead to an underestimate of the turbulent Mach number. Conversely, A781 exhibits a fairly high Mach number $M_{1 \mathrm{D}}=0.22 \pm 0.02$ and $\sigma_{v}=457 \pm 42$. This system does not host a radio halo (Venturi et al. 2011), although residual radio emission at the level of 15-20 mJy at $320 \mathrm{MHz}$ could indicate a steep-spectrum radio halo. One possibility is that this system is in the early stage of a merger and that the turbulent cascade is not fully developed, such that the onset of particle acceleration at the microscale has not yet taken place.

As a word of caution, we note that the conversion between 2D amplitude and turbulent velocity dispersion assumes that all the observed fluctuations are induced by turbulent motions. Additional perturbing phenomena such as unresolved gas clumps or shock fronts will lead to an increase in the fractional amplitude of density perturbations. Although deviating regions such as clear substructures were masked during this analysis, the values reported here might overestimate the intrinsic turbulent Mach number.

Future X-ray imaging spectrometers such as ATHENA (Nandra et al. 2013) or the Hitomi recovery mission XARM will allow us to measure the velocity dispersion directly and will set strong constraints on the models describing the acceleration of relativistic electrons by turbulence in the ICM.

\section{Conclusions}

In this Letter, we presented measurements of the projected amplitude of gas density fluctuations in the ICM at a single scale $k^{-1}=660 \mathrm{kpc}$ and, for the first time, correlated it with the radio emission for a sample of 51 galaxy clusters. Our results can be summarized as follows:

1. We observe a clear segregation between the level of ICM fluctuations between the clusters exhibiting a radio halo and the ones where no radio emission has been detected. The difference between the two populations is significant at the $4.6 \sigma$ level.

2. If the measured fluctuations are interpreted as being entirely due to residual gas motions, the 1D Mach number of turbulent motions is found to be larger in the radio halo populations by about a factor of two compared to the clusters where no radio emission was detected.

3. The turbulent velocity dispersion $\sigma_{v}=M_{3 \mathrm{D}} c_{\mathrm{s}}$ of radio halo systems correlates with the observed radio power (correlation coefficient $0.80 \pm 0.02$ ). The best-fit relation reads $P_{1.4} \propto \sigma_{v}^{3.3 \pm 0.7}$ with $44 \%$ scatter. Thus, the radio power is nearly proportional to the turbulent energy rate $P_{\text {turb }} \propto \sigma_{v}^{3}$.

4. Provided that surface-brightness fluctuations are probing gas motions in the ICM and that the measured turbulence cascades with no break down to the resonant scales where particles can be accelerated, our results corroborate stochastic acceleration via turbulence as the most likely mechanism to boost the emergence of radio halos in galaxy clusters.

We thank the anonymous referee for useful comments. This work was supported in part by NASA Chandra grant
GO7-18121X. M.G. is supported by NASA through Einstein Postdoctoral Fellowship Award Number PF5-160137 issued by the Chandra X-ray Observatory Center, which is operated by the SAO for and on behalf of NASA under contract NAS803060. F.V. acknowledges financial support from the EU Horizon 2020 research and innovation program under the Marie-Sklodowska-Curie grant agreement No. 664931. S.E. acknowledges the financial support from contracts ASI-INAF I/009/10/0, NARO15 ASI-INAF I/037/12/0, and ASI 2015-046-R.0.

\section{References}

Arévalo, P., Churazov, E., Zhuravleva, I., Forman, W. R., \& Jones, C. 2016, ApJ, 818, 14

Arévalo, P., Churazov, E., Zhuravleva, I., Hernández-Monteagudo, C., \& Revnivtsev, M. 2012, MNRAS, 426, 1793

Basu, K. 2012, MNRAS, 421, L112

Brunetti, G., Giacintucci, S., Cassano, R., et al. 2008, Natur, 455, 944

Brunetti, G., \& Lazarian, A. 2011, MNRAS, 412, 817

Brunetti, G., \& Lazarian, A. 2016, MNRAS, 458, 2584

Brunetti, G., Setti, G., Feretti, L., \& Giovannini, G. 2001, MNRAS, 320, 365

Buote, D. A. 2001, ApJL, 553, L15

Cash, W. 1979, ApJ, 228, 939

Cassano, R., Ettori, S., Brunetti, G., et al. 2013, ApJ, 777, 141

Cassano, R., Ettori, S., Giacintucci, S., et al. 2010, ApJL, 721, L82

Cavaliere, A., \& Fusco-Femiano, R. 1976, A\&A, 49, 137

Churazov, E., Vikhlinin, A., Zhuravleva, I., et al. 2012, MNRAS, 421, 1123

Cuciti, V., Cassano, R., Brunetti, G., et al. 2015, A\&A, 580, A97

Dolag, K., Vazza, F., Brunetti, G., \& Tormen, G. 2005, MNRAS, 364, 753

Eckert, D., Molendi, S., Owers, M., et al. 2014, A\&A, 570, A119

Eckert, D., Molendi, S., \& Paltani, S. 2011, A\&A, 526, A79+

Eckert, D., Vazza, F., Ettori, S., et al. 2012, A\&A, 541, A57

Feretti, L., Giovannini, G., Govoni, F., \& Murgia, M. 2012, A\&Ar, 20, 54 Gaspari, M., \& Churazov, E. 2013, A\&A, 559, A78

Gaspari, M., Churazov, E., Nagai, D., Lau, E. T., \& Zhuravleva, I. 2014, A\&A, 569, A67

Hickox, R. C., \& Markevitch, M. 2006, ApJ, 645, 95

Hitomi Collaboration, Aharonian, F., Akamatsu, H., et al. 2016, Natur, 535,117

Hofmann, F., Sanders, J. S., Nandra, K., Clerc, N., \& Gaspari, M. 2016, A\&A, 585, A130

Kalberla, P. M. W., Burton, W. B., Hartmann, D., et al. 2005, A\&A, 440, 775

Kelly, B. C. 2007, ApJ, 665, 1489

Khatri, R., \& Gaspari, M. 2016, MNRAS, 463, 655

Lau, E. T., Kravtsov, A. V., \& Nagai, D. 2009, ApJ, 705, 1129

Macario, G., Venturi, T., Brunetti, G., et al. 2010, A\&A, 517, A43

Mazzotta, P., Rasia, E., Moscardini, L., \& Tormen, G. 2004, MNRAS, 354,10

Miniati, F. 2015, ApJ, 800, 60

Nandra, K., Barret, D., Barcons, X., et al. 2013, arXiv:1306.2307

Petrosian, V., \& Bykov, A. M. 2008, SSRv, 134, 207

Rossetti, M., Eckert, D., Cavalleri, B. M., et al. 2011, A\&A, 532, A123

Schuecker, P., Finoguenov, A., Miniati, F., Böhringer, H., \& Briel, U. G. 2004, A\&A, 426, 387

Smith, R. K., Brickhouse, N. S., Liedahl, D. A., \& Raymond, J. C. 2001, ApJL, 556, L91

Snowden, S. L., McCammon, D., Burrows, D. N., \& Mendenhall, J. A. 1994, ApJ, 424, 714

Snowden, S. L., Mushotzky, R. F., Kuntz, K. D., \& Davis, D. S. 2008, A\&A, 478,615

Thierbach, M., Klein, U., \& Wielebinski, R. 2003, A\&A, 397, 53

Vazza, F., Brunetti, G., Kritsuk, A., et al. 2009, A\&A, 504, 33

Vazza, F., Jones, T. W., Brüggen, M., et al. 2017, MNRAS, 464, 210

Venturi, T., Giacintucci, G., Dallacasa, D., et al. 2011, MNRAS, 414, L65

Venturi, T., Giacintucci, S., Brunetti, G., et al. 2007, A\&A, 463, 937

Venturi, T., Giacintucci, S., Dallacasa, D., et al. 2008, A\&A, 484, 327

Zhuravleva, I., Churazov, E., Arévalo, P., et al. 2015, MNRAS, 450, 4184

Zhuravleva, I., Churazov, E. M., Schekochihin, A. A., et al. 2014, ApJL, 788, L13 